\title{
Cardiac arrest following cardiac tamponade caused by mycosis fungoides malignant pericarditis
}

\author{
Shuku Sato ${ }^{1}$ and Eri Tanaka ${ }^{2}$ \\ ${ }^{1}$ Shonan Kamakura General Hospital \\ ${ }^{2}$ Hayama Heart Center
}

February 25, 2021

\begin{abstract}
MF is a low-grade lymphoma, but on reaching the tumor stage, it can cause cardiac tamponade owing to epicardial infiltration. Myocardial infiltration, even in the absence of abnormal imaging findings, requires attention because it can lead to arrhythmia and cardiac arrest.
\end{abstract}

\section{Title}

Cardiac arrest following cardiac tamponade caused by mycosis fungoides malignant pericarditis

\section{Authors names}

Shuku Sato ${ }^{1)}$, Eri Tanaka ${ }^{2)}$.

\section{Author affiliations}

Division of Hematology, Shonan Kamakura General Hospital

Division of Internal Medicine, Hayama heart center, Kanagawa, Japan

\section{Corresponding author}

Shuku Sato, MD.

Division of Hematology, Shonan Kamakura General Hospital, Kanagawa, in Japan

1370-1 Okamoto, Kamakura, Kanagawa 247-8533 Japan

Telephone: +81-467-46-1717

Facsimile: +81-467-45-0190

E-mail: ocukuhs719@gmail.com

\section{Abstract}

MF is a low-grade lymphoma, but on reaching the tumor stage, it can cause cardiac tamponade owing to epicardial infiltration. Myocardial infiltration, even in the absence of abnormal imaging findings, requires attention because it can lead to arrhythmia and cardiac arrest.

\section{Key clinical Message}

MF is an indolent lymphoma, but when it reaches the tumor stage, it can cause cardiac tamponade due to extracardiac invasion, which may lead to arrhythmia and sudden death due to myocardial invasion. 
Keywords : Mycosis fungoides, cardiac tamponade

A 48-year-old man diagnosed with mycosis fungoides (MF), advanced to the tumor stage (Fig.1a), presented with dyspnea. He was receiving gemcitabine at $1.2 \mathrm{~g} / \mathrm{m}^{2}$ once-weekly for eight weeks. Echocardiography revealed cardiac tamponade caused by malignant pericarditis malignant pericarditis (Fig.1b,c). However, echocardiography, computed tomography, and magnetic resonance imaging showed no evidence of wall thickening or intramyocardial masses suggesting myocardial infiltration. We drained $2 \mathrm{~L}$ pericardial fluid, which showed $\mathrm{T}$ cell clonality by Southern blot analysis, while starting radiation therapy to the pericardium. Thereafter, he developed a complete atrioventricular block, followed by cardiac arrest. In this case, MF might have invaded the myocardium and disordered the impulse conduction system. Malignant lymphoma (ML) occasionally presents with cardiac involvement, but most patients have no suggestive symptoms, and diagnosis while alive is difficult ${ }^{1}$. William et al. reported that out of 48 autopsies of ML cases with cardiac involvement, only $37 \%$ involved the epicardium only, while the rest involved the ventricular wall and epicardium $^{2}$. MF is a low-grade lymphoma, but on reaching the tumor stage, it can cause cardiac tamponade owing to epicardial infiltration. Myocardial infiltration, even in the absence of abnormal imaging findings, requires attention because it can lead to arrhythmia and cardiac arrest.

\section{Conflict of interest}

All authors declare no conflicts of interest.

\section{Informed consent}

Written informed consent was obtained from the patient.

\section{Author contributions}

SS and ET: participated in the management of this patient. SS: prepared and edition of this manuscript.

\section{References:}

1) Rosemberg SA, Diamond HD, Jaslowitz B, Craver LF. Lymphosarcoma : A review of 1269 cases. Medicine. 1961;40:31-84.

2) Roberts WC, Glancy DL, DeVita Jr VT. Heart in malignant lymphoma (Hodgkin's disease, lymphosarcoma, reticulum cell sarcoma and mycosis fungoides). A study of 196 autopsy cases. Am J Cardiol. 1968;22:85-107.

\section{Hosted file}

Figure \begin } \{ \mathrm { CJK } \} \{ \mathrm { UTF } 8 \} \{ \text { gbsn } \} \text { . \end } \{ \mathrm { CJK } \} \backslash \text { selectlanguage\{english\}pdf available at https: } //authorea.com/users/398157/articles/510821-cardiac-arrest-following-cardiac-tamponadecaused-by-mycosis-fungoides-malignant-pericarditis 